\title{
The COP9 Signalosome Is Required for Light-Dependent Timeless Degradation and Drosophila Clock Resetting
}

\author{
Alyson Knowles, ${ }^{1}$ Kyunghee Koh, ${ }^{2}$ June-Tai Wu, ${ }^{3}$ Cheng-Ting Chien, ${ }^{3}$ Daniel A. Chamovitz, ${ }^{4}$ and Justin Blau ${ }^{1}$ \\ ${ }^{1}$ Department of Biology, New York University, New York, New York 10003, ${ }^{2}$ Department of Neuroscience, University of Pennsylvania, Philadelphia, \\ Pennsylvania 19104, ${ }^{3}$ Institute of Molecular Biology, Academia Sinica, Taipei 115, Taiwan, and ${ }^{4}$ Department of Plant Sciences, Tel Aviv University, Tel Aviv \\ 69978, Israel
}

The ubiquitin-proteasome system plays a major role in the rhythmic accumulation and turnover of molecular clock components. In turn, these $\sim 24 \mathrm{~h}$ molecular rhythms drive circadian rhythms of behavior and physiology. In Drosophila, the ubiquitin-proteasome system also plays a critical role in light-dependent degradation of the clock protein Timeless (TIM), a key step in the entrainment of the molecular clocks to light-dark cycles. Here, we investigated the role of the COP9 signalosome (CSN), a general regulator of protein degradation, in fly circadian rhythms. We found that null mutations in the genes encoding the CSN4 and CSN5 subunits prevent normal TIM degradation by light in the pacemaker lateral neurons (LNs) as does LN-specific expression of a dominant-negative CSN5 transgene. These defects are accompanied by strong reductions in behavioral phase shifts of adult flies lacking normal CSN5 activity in LNs. Defects in TIM degradation and resetting of behavioral phases were rescued by overexpression of Jetlag (JET), the F-box protein required for light-mediated TIM degradation. Flies lacking normal CSN activity in all clock neurons are rhythmic in constant light, a phenotype previously associated with jet mutants. Together, these data indicate that JET and the CSN lie in a common pathway leading to light-dependent TIM degradation. Surprisingly, we found that manipulations that strongly inhibit CSN activity had minimal effects on circadian rhythms in constant darkness, indicating a specific role for the CSN in light-mediated TIM degradation.

Key words: circadian rhythms; COP9 signalosome; Timeless; protein degradation; light signaling; entrainment

\section{Introduction}

Circadian $(\sim 24 \mathrm{~h})$ rhythms help organisms anticipate daily environmental changes and persist even in constant environments. These behavioral and physiological rhythms are controlled by intracellular molecular clocks that are based on transcription/ translation negative-feedback loops that are also highly regulated by posttranslational modifications (for review, see Allada et al., 2001).

In Drosophila, two of the essential clock genes period ( per) and timeless (tim) are rhythmically expressed and their protein products are posttranslationally regulated. Phosphorylation of PER and TIM proteins regulates their subcellular localization and stability (for review, see Hardin, 2005). Phosphorylated PER is targetted by Slimb, the F-box protein subunit of an E3 ubiquitin

Received Jan. 30, 2008; revised Dec. 19, 2008; accepted Dec. 22, 2008.

This work was supported by a National Alliance for Research on Schizophrenia and Depression Young Investigator Award (K.K.) and National Institutes of Health Grant GM063911 (J.B.). This investigation was mainly conducted in a facility constructed with support from Research Facilities Improvement Grant C06 RR-15518-01 from the National Center for Research Resources-National Institutes of Health. We are very grateful to Jae Park, Michael Rosbash, Danny Segal, Amita Sehgal, and Mike Young for fly strains and antibodies. We also thank Ben Collins and Claude Desplan for constructive comments throughout the course of this work, Simon Sprecher for help in analyzing the larval visual system, Mark Siegal for advice on statistics, and Ben Collins and Tricia McKenzie for comments on this manuscript.

Correspondence should be addressed to Justin Blau, Department of Biology, New York University, 100 Washington Square East, New York, NY 10003. E-mail: justin.blau@nyu.edu.

J.-T. Wu's present address: Department of Medical Research, National Taiwan University Hospital, Taipei 100, Taiwan.

DOI:10.1523/JNEUROSCI.0429-08.2009

Copyright $\odot 2009$ Society for Neuroscience $\quad$ 0270-6474/09/291152-11\$15.00/0 ligase complex for PER ubiquitination and proteosomal degradation (Grima et al., 2002; Ko et al., 2002). Two F-box proteins contribute to TIM degradation: Slimb is required for TIM degradation in constant darkness (DD) (Grima et al., 2002), whereas Jetlag (JET) (Koh et al., 2006; Peschel et al., 2006) is required for rapid TIM degradation by light, which in turn entrains the Drosophila molecular clock to light-dark (LD) cycles (Hunter-Ensor et al., 1996; Lee et al., 1996; Myers et al., 1996; Zeng et al., 1996). Clock cells receive light cell autonomously via the Cryptochrome photoreceptor (CRY) and also from the visual system (Stanewsky et al., 1998). Photoreception via CRY results in TIM phosphorylation (Naidoo et al., 1999) and allows recognition by JET. However, there are likely to be unidentified components required for Slimb- and JET-mediated TIM degradation. To identify novel elements, we took a leaf from Arabidopsis and tested a role for the COP9 signalosome (CSN) in Drosophila circadian rhythms.

The CSN was identified in Arabidopsis as a regulator of lightdependent growth (Wei et al., 1994; Chamovitz et al., 1996). Arabidopsis CSN is required for degradation of the transcription factor HY5 under dark conditions, whereas blue-light signals, transduced via the Arabidopsis Cry1 photoreceptor, stabilize HY5 (Osterlund et al., 2000; Harari-Steinberg et al., 2001). Thus, Arabidopsis and Drosophila share regulation of protein stability via CRY.

The CSN is an eight protein complex (Deng et al., 2000; Wei and Deng, 2003) that removes Nedd8, a ubiquitin-like modifier, from the Cullin subunit of Cullin-based E3-ubiquitin ligases 
A
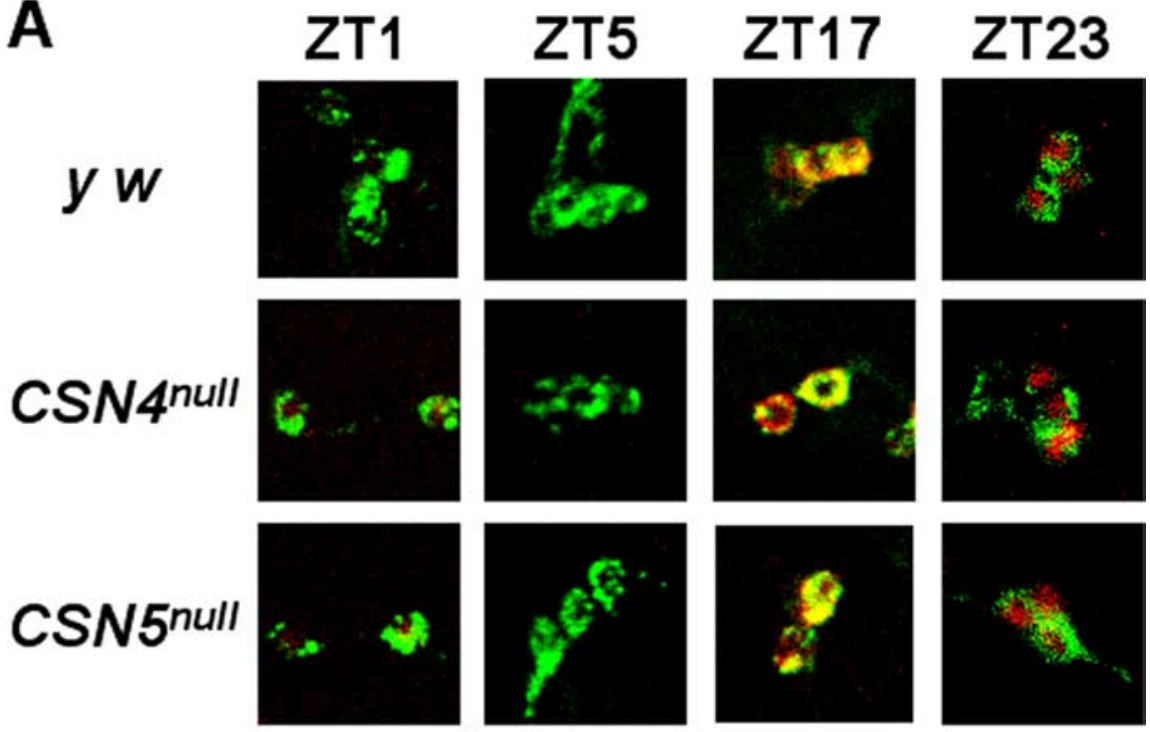

B
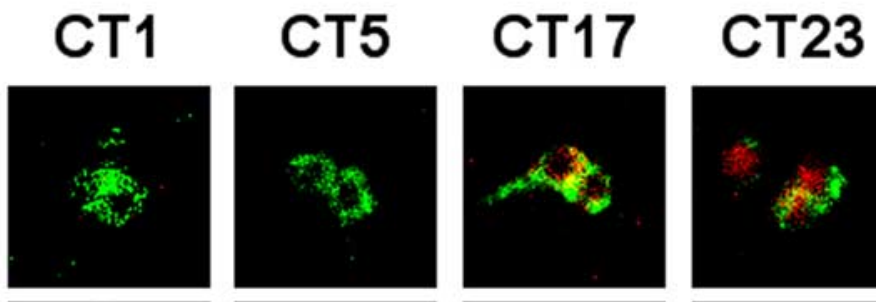

CSN4null
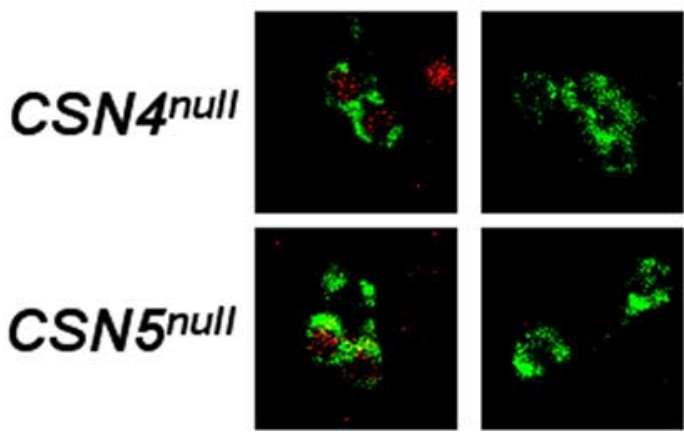
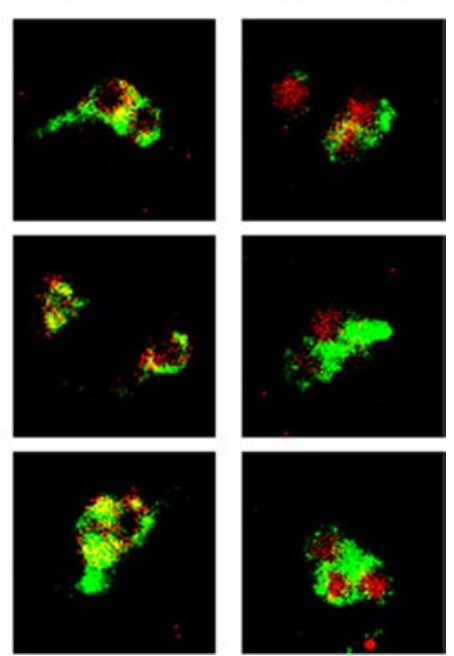

Figure 1. Rhythms in TIM protein levels in LD and DD in CSN4 ${ }^{\text {null }}$ and CSN5 $5^{\text {null }}$ mutant larval LNs. $\boldsymbol{A}, \boldsymbol{B}$, TIM levels (red) were measured in LD cycles at four time points (ZT1, ZT5, ZT17, and ZT23) (A) and in DD at four time points (CT1, CT5, CT17, and CT23) $(\boldsymbol{B})$ in the LNs of $y$ w (top panels), CSN4 ${ }^{\text {null }}$ (middle panels), and CSN5 ${ }^{\text {null }}$ larvae (bottom panels). LNs were marked using an antibody specific for PDF (green). The images shown are representative of at least five brains stained per time point in at least three independent experiments. The time points shown were taken from separate experiments, although each experiment included all genotypes.

(CRLs) (Lyapina et al., 2001; Cope et al., 2002; Wolf et al., 2003). The prototypical CRLs are the SCF-type E3 ligases. SCF complexes consist of the linker protein Skp1, the scaffold protein Cul1, a RING-finger protein (Rbx), which recruits the E2 Ubiquitin-conjugating enzyme, and an F-box protein that recruits substrates. SCF complexes are activated to ubiquitinate substrates by covalent attachment of Nedd 8 to Cul1, and inactivated by the deneddylase activity of CSN subunit 5 (CSN5) (Cope et al., 2002). The CSN also mediates phosphorylation and deubiquitination of ubiquitin-proteasome pathway substrates (for review, see Harari-Steinberg and Chamovitz, 2004).

SCF complexes have been broadly implicated in circadian rhythms: in Drosophila, SCF ${ }^{\text {Slimb }}$ regulates PER stability in LD and DD, and TIM stability in DD, whereas $\mathrm{SCF}^{\mathrm{JET}}$ regulates TIM

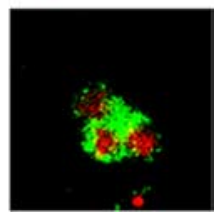

stability in LD; in mammals, $\mathrm{SCF}^{\mathrm{Fbxl3}}$ regulates mCry protein stability (Busino et al., 2007; Godinho et al., 2007; Siepka et al., 2007); and in Neurospora, SCF complexes containing the F-box protein FWD-1 $\left(\mathrm{SCF}^{\mathrm{FWD-1}}\right)$ regulate stability of the clock protein Frequency (FRQ) (He et al., 2003). Furthermore, a role for the CSN in circadian rhythms has been demonstrated in csn-2 null mutant Neurospora (He et al., 2005). Although CSN inactivation might be expected to lead to constitutively active SCF complexes and reduced substrate levels, FRQ overaccumulates in csn-2 null mutants. This is presumably because constitutive activation of $\mathrm{SCF}^{\mathrm{FWD}-1}$ leads to degradation of the FWD-1 F-box protein itself rendering FRQ stable despite being hyperphosphorylated.

Here, we tested the role of the CSN in Drosophila circadian rhythms. We found that the CSN is required cell autonomously in circadian pacemaker neurons for rapid light-mediated TIM degradation and behavioral phase shifts, but not for molecular oscillations per se. The phenotypes caused by inhibiting CSN activity in pacemaker neurons were rescued by overexpressing jet. Furthermore, flies with reduced CSN activity in all clock neurons are rhythmic in constant light (LL), a phenotype also exhibited by jet and cry mutants. These results indicate that the CSN mediates $\mathrm{SCF}^{\mathrm{JET}}$, but not $\mathrm{SCF}^{\text {Slimb }}$, control of TIM stability such that the CSN is required for light-mediated TIM degradation, but not for the general maintenance of circadian rhythms.

\section{Materials and Methods}

Fly strains. The CSN $4^{\text {null }}$ and $C S N 5^{\text {null }}$ mutant strains have been described previously (Freilich et al., 1999; Oron et al., 2002). PCR analysis determined that CSN $4^{\text {null }}$ and CSN $5^{\text {null }}$ mutant larvae and $y w$ control larvae carry the $l s$-tim allele (data not shown), ruling out genetic variations in tim as an explanation for the reduced light sensitivity of TIM in CSN4 $4^{\text {null }}$ and CSN5 $^{\text {null }}$ larvae (Peschel et al., 2006). For expression in lateral neurons (LNs), we used the $P d f_{0.5}$-Gal4 driver (Park et al., 2000). Other Gal4 drivers used were tim-Gal4 (Emery et al., 1998) and tubulinP-Gal4 (Lee and Luo, 1999). Pdf-Gal80 flies were described by Stoleru et al. (2004). Flies carrying wild-type and dominant-negative CSN5 transgenes (UAS-CSN5 and UAS-CSN5 ${ }^{D N}$, respectively) were also described previously (Wu et al., 2005) as were UAS-jet flies (Koh et al., 2006). The $U A S-C S N 5^{D N 2}$ chromosome also carried the $C S N 5^{\text {null }}$ allele, so larvae or flies carrying this transgene had only one wild-type CSN5 allele. Sequencing the jet locus in tim-Gal4 and UAS-CSN $5^{D N 2}$ flies revealed that they both have wild-type jet, ruling out variation in jet as a contributor to rhythmicity in constant light in flies with tim-Gal4 and UAS-CSN5 $5^{D N 2}$ transgenes (Peschel et al., 2006).

TIM degradation assays and quantitation. The yellow white $(y w)$ strain served as controls in most TIM degradation assays. All Drosophila strains were entrained to $12 \mathrm{~h} \mathrm{LD}$ cycles for at least $3 \mathrm{~d}$ before 

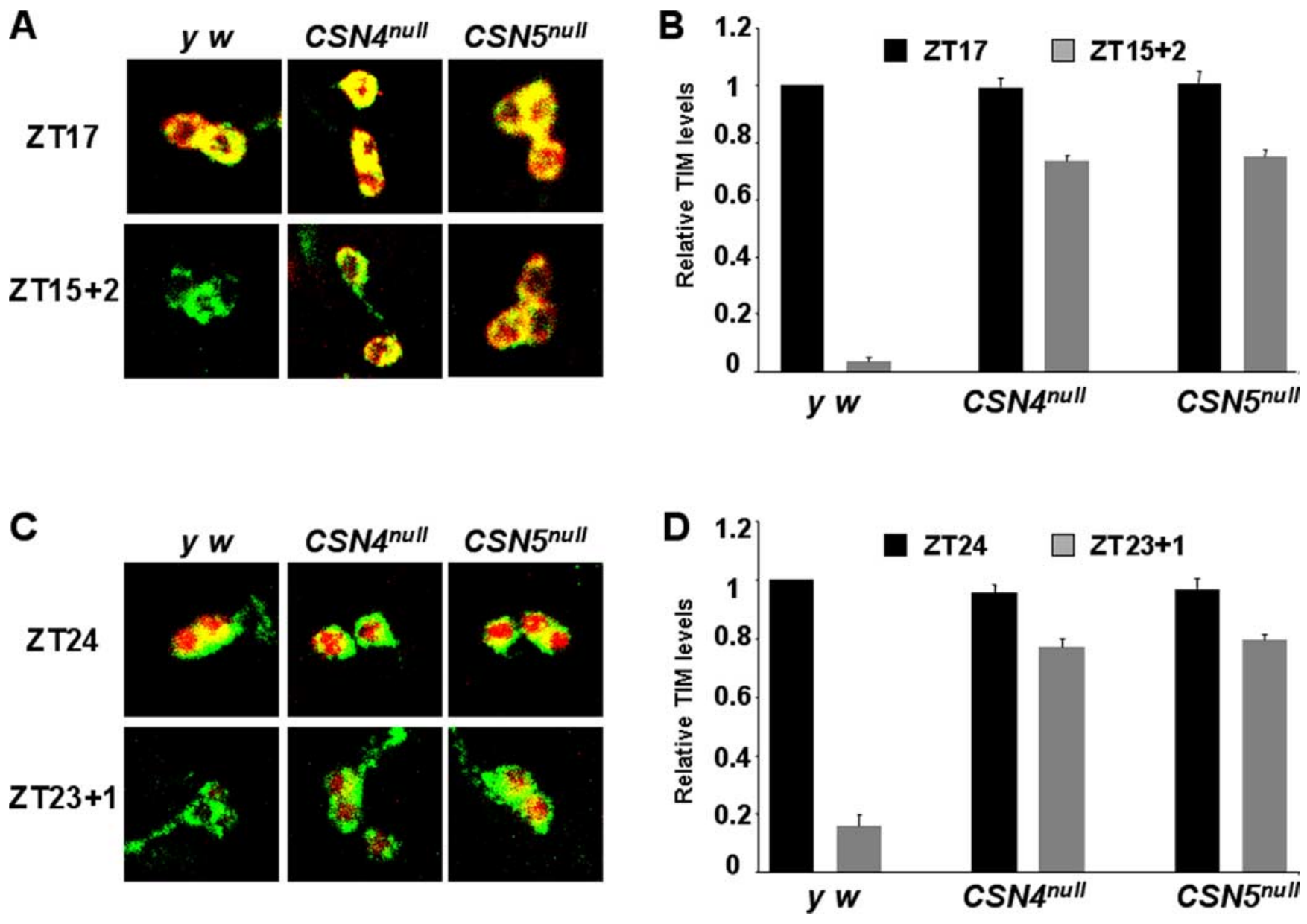

Figure 2. CSN4 and CSN5 are required for normal light-mediated TIM degradation in larval LNs. A, C, TIM degradation by light in LNs was measured by exposing larvae to $\sim 750$ lux light for either $2 \mathrm{~h}$ starting at ZT15 (A) or $1 \mathrm{~h}$ starting at ZT23 $(\boldsymbol{C})$ and then compared with the equivalent unpulsed time point (ZT17 for $\boldsymbol{A} ; \mathrm{ZT24}$ for $\boldsymbol{C}$. LNs from unpulsed larvae are shown in the top panels, and light-exposed larvae are shown in the bottom panels. Brains were stained with TIM (red) and PDF (green) as in Figure 1. The images on the left are representative of at least six brains stained per genotype per condition in at least eight independent experiments. Quantitation was performed for three of these experiments using at least six brains per genotype. $\boldsymbol{B}, \boldsymbol{D}$, TIM levels in LNs were quantitated (see Materials and Methods) with unpulsed levels in black and light-exposed levels in gray (B, D). TIM levels at ZT17 or ZT23 in y $w$ control larvae were set to 1 , and other genotypes were normalized to this. Error bars show SEM. TIM is degraded by light significantly more in $y$ w larvae compared with CSN4 ${ }^{\text {null }}$ and CSN5 ${ }^{\text {null }}$ larvae at each time point $(p<0.001)$.

conducting an experiment. In the experiments in Figures 1-3, LDentrained third-instar $y w, C S N 4^{\text {null }}, C S N 5^{\text {null }}$, and $P d f>C S N 5$, $C S N 5^{\text {null }}$ larvae were collected on grape juice plates rather than in vials. In these experiments, homozygous CSN4 $4^{\text {null }}$ and CSN5 $5^{\text {null }} \mathrm{mu}$ tants were separated from heterozygous $C S N 4^{\text {null }} / T(I I ; I I I)$ and $C S N 5^{\text {null }} /$ TM6b larvae, respectively, by the absence of the Tubby marker. For each TIM degradation experiment, 10-12 third-instar larval brains per genotype were dissected at the assigned experimental time points. For the adult TIM degradation experiments at zeitgeber time 21 (ZT21; time in LD), five to eight brains were analyzed per genotype in three independent experiments. Immunostainings for TIM and pigment-dispersing factor (PDF) were performed as previously described using TIM antibodies generously provided by $\mathrm{M}$. Young (Rockefeller University, New York, NY) and A. Sehgal (University of Pennsylvania, Philadelphia, PA) and a monoclonal antibody to PDF (Cyran et al., 2005). Images were captured by confocal microscopy.

For quantitation of TIM levels, ImageJ software was used to measure the average TIM staining intensity in each LN (detected via PDF staining) and the background signal was subtracted. The average TIM level per genotype per time point was calculated using between 10-20 LNs from 5 to 10 brains (except only 5 LNs from 3 brains for UAS-CSN $5^{D N 2}$ ). Cells lying so close together that their borders could not be distinguished were not included in the analysis. The average TIM staining intensity of unpulsed control $(y w)$ larvae was designated as 1, and the average TIM intensity for the remaining genotypes and conditions was normalized to this value. TIM levels from three independent experiments were averaged together. Statistical differences in TIM levels between genotypes were determined using an unpaired $t$ test.

Behavioral analysis. Young male flies were entrained to LD cycles at $25^{\circ} \mathrm{C}$ and loaded into Trikinetics locomotor activity monitors. For light pulse experiments, flies were given a $2 \mathrm{~min}, 750$ lux light pulse at either ZT15 or ZT21 during the dark phase of the fourth day in LD, and then transferred to DD. For constant light experiments, flies were entrained to LD cycles for 2-3 d, and then assayed in 100 lux constant light for $6 \mathrm{~d}$. This light level is lower than used previously to detect rhythmicity in jet ${ }^{r}$ mutants (Koh et al., 2006) and was chosen because preliminary data suggested that the constant light phenotype of $C S N 5^{D N 2}$ expressing flies was not as strong as in jet ${ }^{r}$ mutants.

Behavioral data were analyzed using the ClockLab program running on MatLab to calculate the $\chi^{2}$ period length. Power was calculated by measuring the height of the highest amplitude peak. Flies with amplitudes below the significance line were scored as arrhythmic as were flies given a low-amplitude power if a clear rhythm could not be seen on visual inspection. For the phase shift experiments, the offset of activity during the first $4 \mathrm{~d}$ in DD was measured using ClockLab. These numbers were then averaged and used to compare light-pulsed and unpulsed flies to generate the average phase shift per genotype per condition. Flies displaying arrhythmic behavior or unclear offsets of activity were not used in the analysis. Between 10 and 70 flies per genotype were used each for each 
A

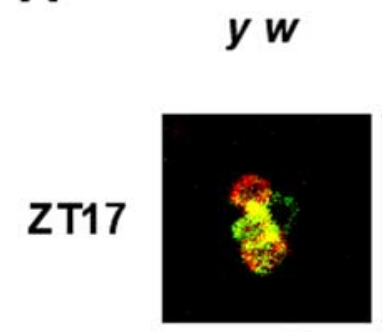

$\mathrm{ZT} 15+2$
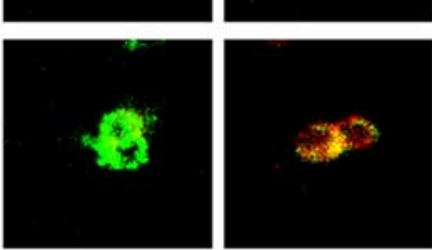

CSN5
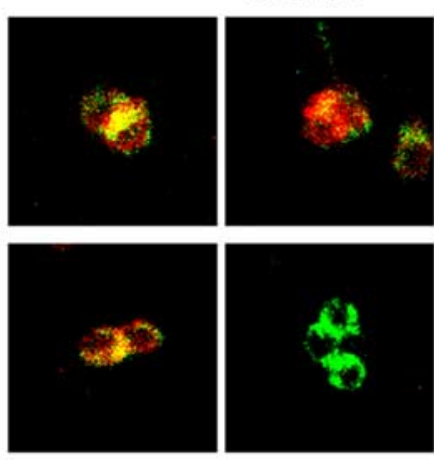

Pdf >

CSN5 ${ }^{W T}$; CSN5 $5^{\text {null }}$

C
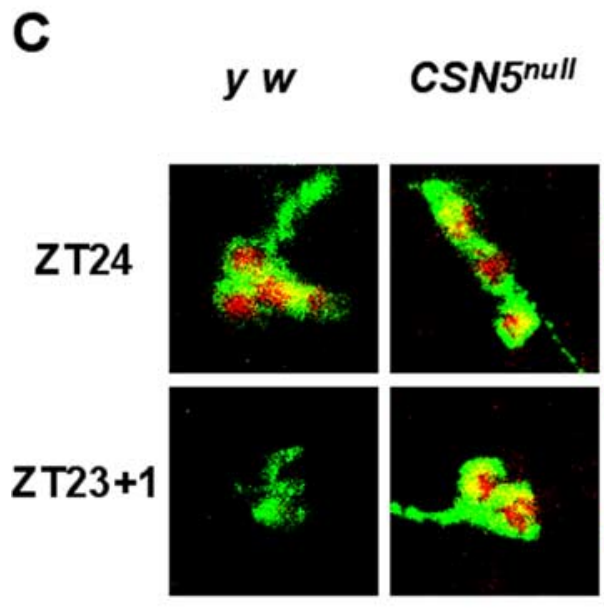

Pdf > CSN5 $^{W T}$; CSN5
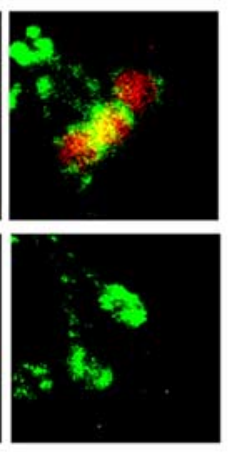

B

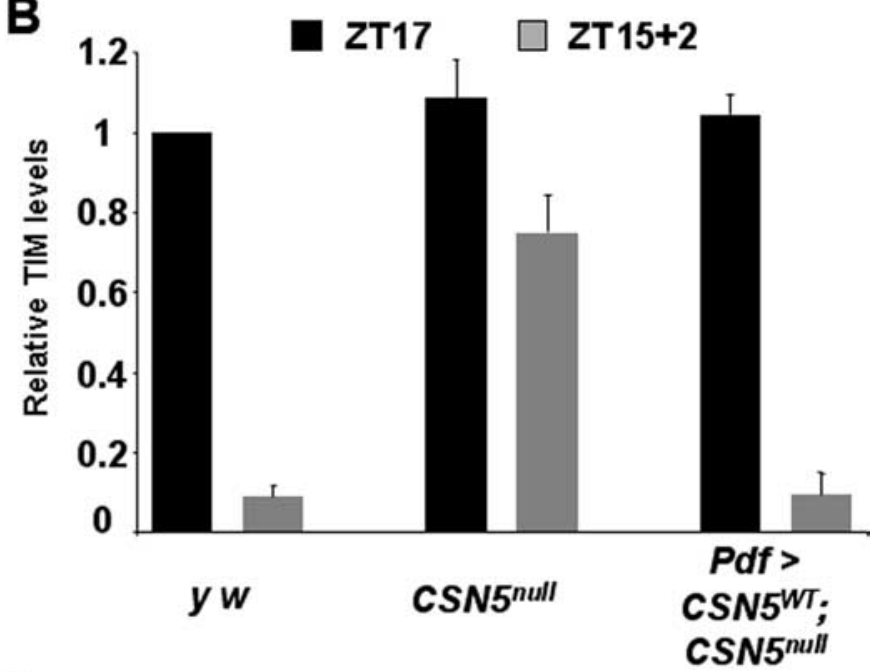

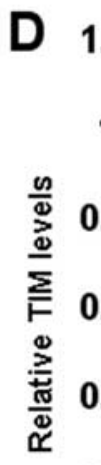

0.2

$\mathbf{0}$

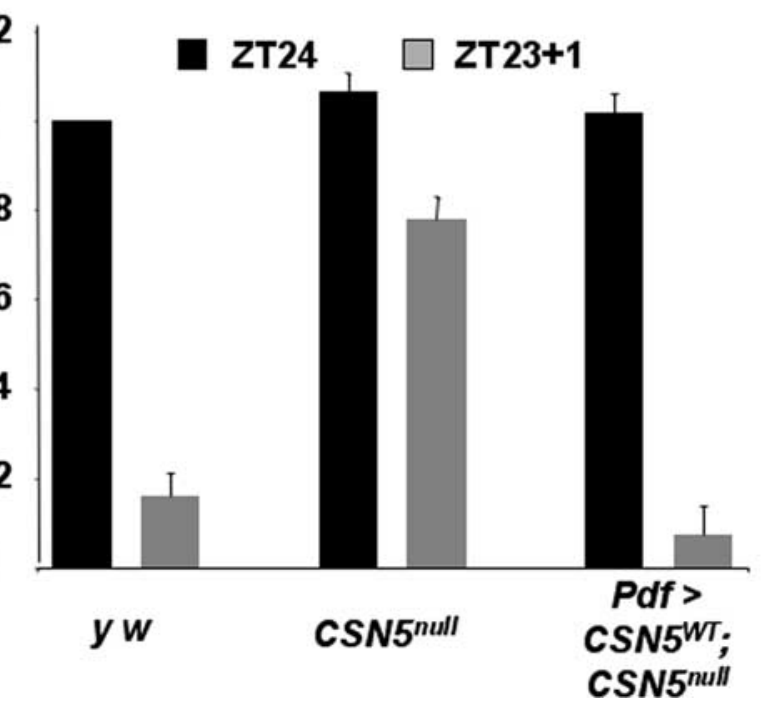

Figure 3. CSN5 is required in larval LNs for normal light-dependent TIM degradation. To test whether CSN5 acts cell autonomously in light-mediated TIM degradation, $P d f_{0.5}$-Gal4 was used to restore CSN5 expression specifically to larval LNs using a UAS-CSN5 transgene in a CSN5 ${ }^{\text {null }}$ background (Pdf $>$ CSN5 ${ }^{W T}$, CSN5 $5^{\text {null }}$; right panels). $\boldsymbol{A}-\boldsymbol{D}$, tIM levels were measured as in Figure 2 for light pulses at ZT15 $(\boldsymbol{A}, \boldsymbol{B})$ or ZT23 $(\boldsymbol{C}, \boldsymbol{D})$. The images on the left are representative of at least six brains stained per time point in at least five independent experiments. Quantitation was performed for three of these experiments using at least five brains per genotype. Error bars show SEM. The quantitation on the right reveals that light degrades TIM in Pdf $>$ CSN $5^{W T}$, CSN $5^{n u l l}$ larvae significantly more than in $\left(S N 5^{\text {null }}\right.$ larvae $(p<0.001)$, and not different from $y$ w larvae $(p>0.1)$.

light pulse and the results are a combination of two to six independent experiments. Flies heterozygous for $P d f_{0.5}-$ Gal4 transgenes on the second and third chromosomes served as the behavioral control in the phase shift experiments. Statistical differences in phase shifts were determined using a Wilcoxon two-sample test. For constant light experiments, all flies were assigned a power value even if there was no obvious peak above the significance line (i.e., they were arrhythmic). For these flies, the power was measured at a local peak between 23 and $27 \mathrm{~h}$. This was done to allow a numerical comparison between the overall strength of the rhythm between different genotypes for all of the flies tested. Unpaired $t$ tests (assuming unequal variance) were used to compare the power of the rhythm between genotypes.

\section{Results}

The molecular clock is functional in CSN4 and CSN5 mutant pacemaker neurons

To test for a role of the CSN in the Drosophila molecular clock, we initially tested whether the clock is functional in null mutants in the genes encoding the CSN4 and CSN5 subunits (CSN $4^{\text {null }}$ and CSN5 $^{\text {null }}$ ) (Freilich et al., 1999; Oron et al., 2002). These mutants affect the integrity of the CSN complex differently: In CSN4 $4^{\text {null }}$ mutants, no CSN complex is detected, although individual subunits, such as CSN5 and CSN7, are present in smaller complexes; whereas in $\mathrm{CSN}^{\text {null }}$ mutants, the remaining CSN subunits still form a complex that maintains some function (Oron et al., 2002, 2007). Although essential, Drosophila mutants homozygous for either $C S N 4^{\text {null }}$ or $C S N 5^{\text {null }}$ survive to third-instar larvae, because of maternally deposited CSN protein (Oron et al., 2002), at which point development arrests. Because third-instar larvae have functional pacemaker neurons capable of directing a simple circadian behavior (Mazzoni et al., 2005), we analyzed the effect of the CSN $4^{\text {null }}$ and $C S N 5^{\text {null }}$ mutations in larvae.

We used antibodies to TIM protein to test for oscillations of the molecular clock in larval pacemaker LNs, which were localized with an antibody against the PDF neuropeptide. In control $(y w)$ larvae in LD cycles, TIM is first detected in the cytoplasm of LNs after the lights go off (ZT17) (Fig. 1A). Later in the night, TIM is found in the nucleus (ZT23) (Fig. 1A) and is then undetectable after lights on (ZT1 and ZT5) (Fig. 1A) because TIM is light sensitive. Control larvae exhibit a similar pattern of TIM 
A
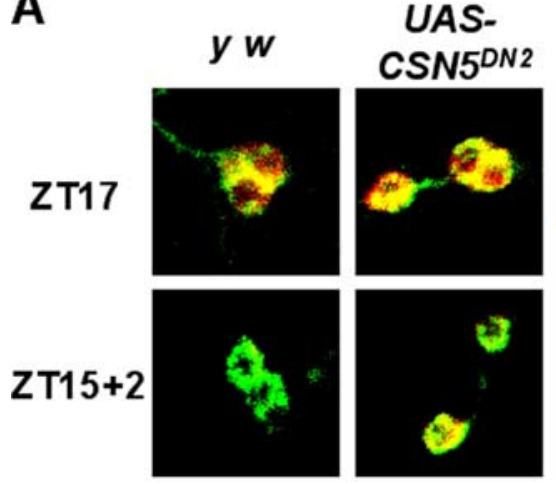

C

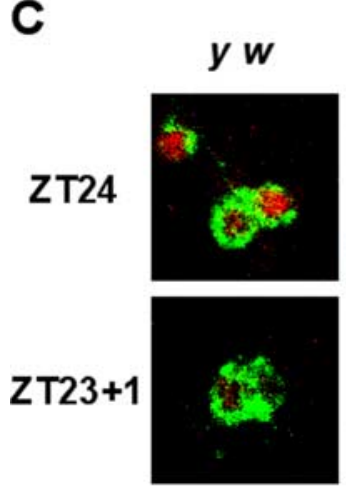

UASCSN5 $5^{D N 2}$
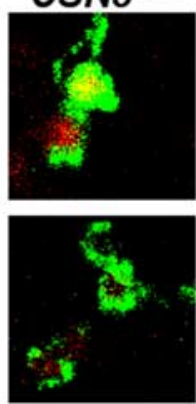
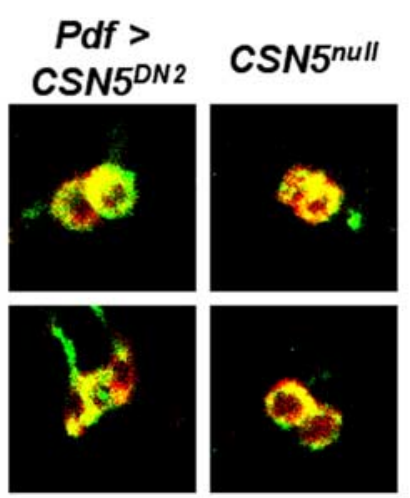

Pdf $>$ CSN5 $5^{\text {DN2 }}$
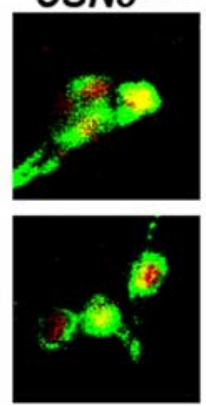
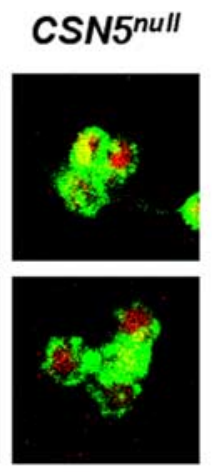

B

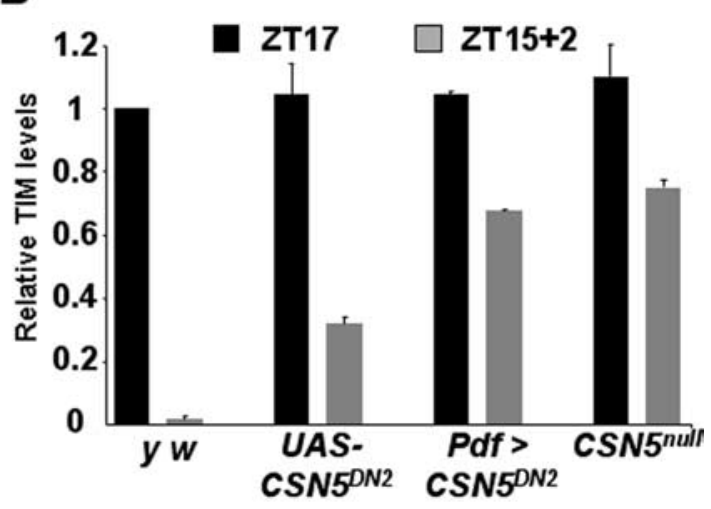

D

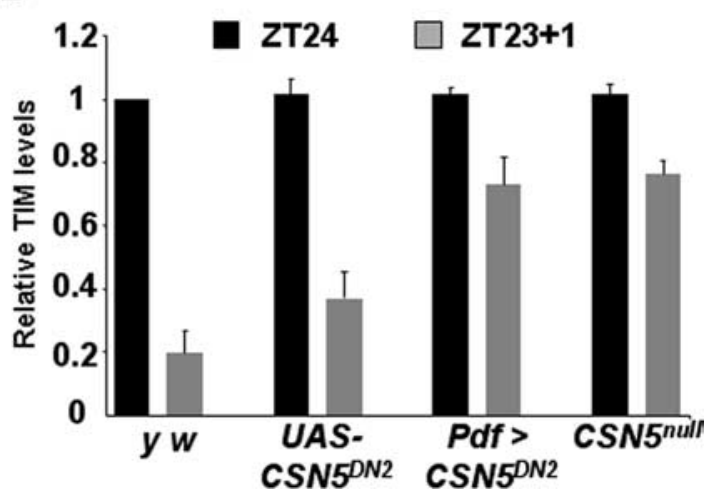

Figure 4. Expression of a dominant-negative CSN5 transgene in larval LNs blocks normal TIM degradation. $\boldsymbol{A}-\boldsymbol{D}$, The dominant-negative UAS-CSN5 ${ }^{D N 2}$ transgene was expressed via Pdf 0.5 -Gal4 $\left(P d f>C^{2 N N}{ }^{D N 2}\right)$ and TIM degradation measured as in Figure 2 with light pulses starting at ZT15 $(\boldsymbol{A}, \boldsymbol{B})$ and ZT23 (C, D). TIM levels (red) in LNs of Pdf $>$ CSN5 $5^{\text {DN2 }}$ larvae were compared with control $y w$ and UAS-CSN5 ${ }^{D N 2}$ larvae (two left panels) and CSN5 $5^{\text {null }}$ mutant larvae (right panels). The images on the left are representative of at least five brains stained per time point in at least four independent experiments. Quantitation was performed for three of these experiments using at least five brains per genotype, except the UAS-CSN5 $5^{\text {DN2 }}$ control which had at least three brains per experiment. Error bars show SEM. The quantitation reveals that levels of TIM degradation are similar in $P d f>C S N 5^{D N 2}$ and (SN5 ${ }^{\text {null }}$ larvae $(~ p>0.1)$. Levels of TIM degradation are significantly less in $P d f>C S N 5^{D N 2}$ and $C S N 5^{\text {null }}$ larvae than in $y$ w larvae $(p<0.001)$.

Table 1. Locomotor activity rhythms of UAS-CSN5 and UAS-jet transgenic flies in constant darkness

\begin{tabular}{|c|c|c|c|}
\hline Genotype & \% Rhythmic ( $n=$ total tested) & Period (h) \pm SEM & Power \pm SEM \\
\hline Pdf-Gal4/+;Pdf-Gal4/+ & $81(75)$ & $23.9 \pm 0.1$ & $515 \pm 16$ \\
\hline Pdf-Gal4 / +;Pdf-Gal4/UAS-CSN5 ${ }^{W T}$ & $87(52)$ & $23.9 \pm 0.1$ & $653 \pm 21$ \\
\hline tim-Gal $4 /+;$ UAS-CSN5 ${ }^{W T} /+$ & $71(30)$ & $23.8 \pm 0.1$ & $552 \pm 31$ \\
\hline Pdf-Gal4/UAS-CSN5 ${ }^{\text {DN4 }} ;$ Pdf-Gal4 $/+$ & $88(30)$ & $23.8 \pm 0.1$ & $478 \pm 35$ \\
\hline tim-Gal4 / +;UAS-CSN5 $5^{\text {DN2 }}, \operatorname{CSN}^{\text {null }} /+$ & $70(28)$ & $23.7 \pm 0.1$ & $495 \pm 23$ \\
\hline tim-Gal4 / UAS-CSN5 ${ }^{D N 4}$ & $71(30)$ & $23.7 \pm 0.1$ & $598 \pm 28$ \\
\hline tim-Gal4 / +;UAS-jet / + & $85(34)$ & $24.1 \pm 0.1$ & $618 \pm 30$ \\
\hline Pdf-Gal4 / UAS-CSN5 ${ }^{\text {DN4 }}$; Pdf-Gal4 / UAS-jet & $97(66)$ & $24.1 \pm 0.1$ & $765 \pm 24$ \\
\hline tim-Gal4 / UAS-CSN5 ${ }^{\text {DN4 }}$; UAS-jet / + & $75(44)$ & $23.9 \pm 0.1$ & $497 \pm 12$ \\
\hline
\end{tabular}

The period for each fly across $8 \mathrm{~d}$ in DD was determined by $\chi^{2}$ analysis. Power was calculated using ClockLab with 5 min collection bins. Flies deemed arrhythmic by ClockLab were excluded from average power calculations.

cytoplasmic accumulation, nuclear entry, and degradation in LNs during the first day of DD after entrainment to LD cycles (Fig. $1 B$ ).

The profile of TIM protein in CSN $4^{\text {null }}$ and CSN5 $5^{\text {null }}$ mutant larvae was very similar to the wild-type controls. The only difference was that low levels of TIM were detectable in the nucleus in the early morning in LD cycles in the mutants (ZT1) (Figure 1A). TIM was undetectable in the mutants by ZT5 indicating that TIM can still be degraded in CSN $4^{\text {null }}$ and CSN $5^{\text {null }}$ mutants. Similarly, TIM could also be detected at low levels in DD at circadian time 1 (CT1; time in DD), but not at CT5.
The cycle of cytoplasmic TIM accumulation, nuclear translocation, and turnover is functional in these mutants, indicating that overall their molecular clocks are functional. Because no deneddylase activity can be detected in either CSN $4^{\text {null }}$ or CSN5 $^{\text {null }}$ mutant larvae (Oron et al., 2007), our results suggest that the CSN is not essential for circadian cycling of Drosophila clock proteins. Consistent with this idea, we also found that circadian rhythms of adult locomotor activity are unaffected when a potent dominant-negative CSN5 transgene is expressed in adult clock neurons (Table 1) (discussed in detail later). 

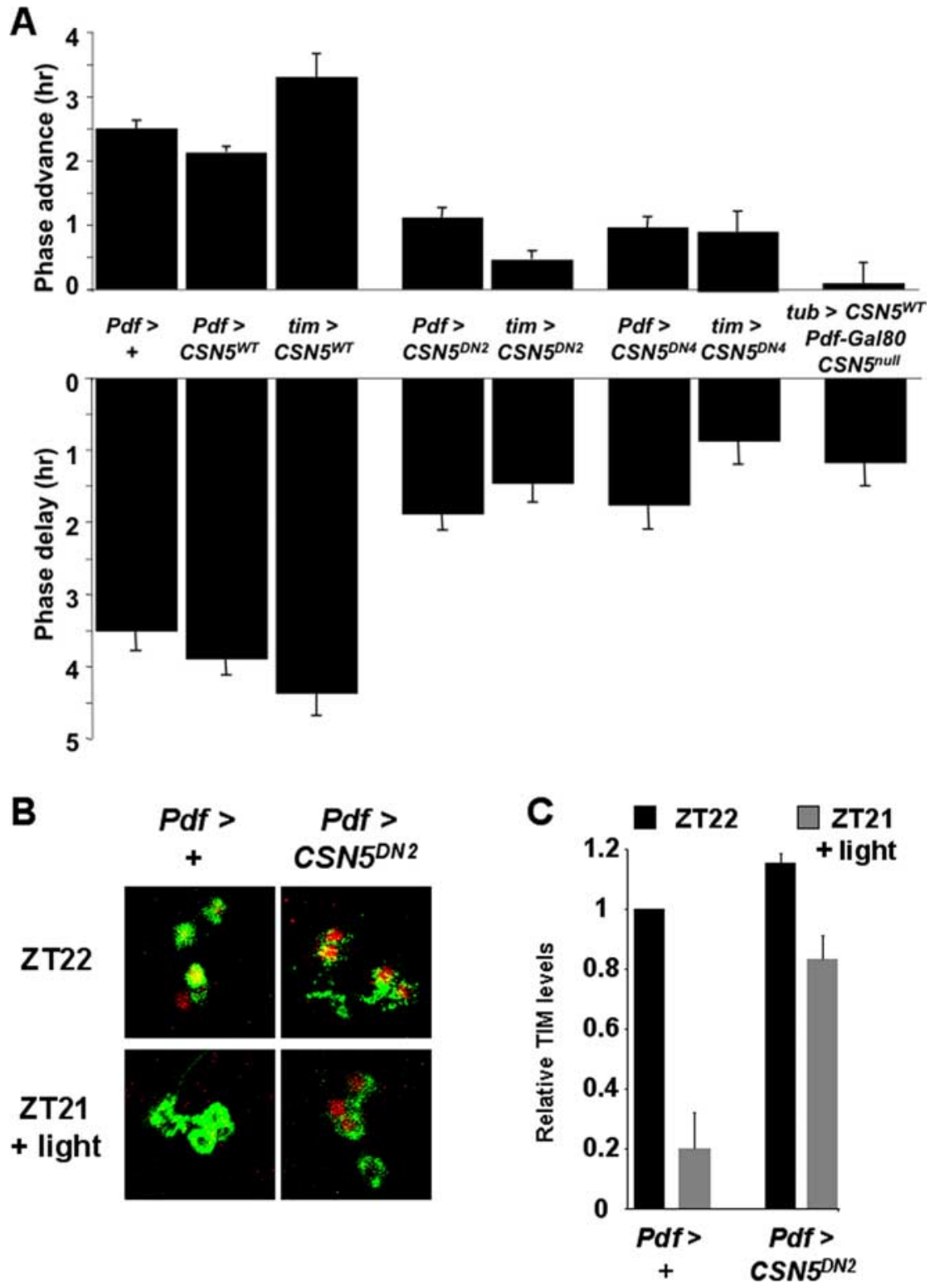

Figure 5. CSN5 is required for normal phase resetting of adult behavioral rhythms and TIM degradation by light. $A$, Flies entrained to $12 \mathrm{~h} \mathrm{LD}$ cycles for at least $4 \mathrm{~d}$ were given a 2 min light pulse of $\sim 750$ lux ate ither ZT15 (phase delays, bottom) or ZT21 (phaseadvances, top) and shifted to DD. We used flies heterozygous for two copies of the $P d f_{0,5}$-Gal 4 driver as controls ( $P d f>+$ ). Flies analyzed had either a wild-type UAS-CSN5 transgene or one of two UAS-CSN5 dominant-negative transgenes (UAS-CSN5 ${ }^{D N 2}$ or UAS-CSN5 ${ }^{D N 4}$ ). The transgenes were expressed using two copies of the $P d f f_{0.5}-$ Gal 4 driver (Pdf $>C S N 5 ;$ Pdf $>C S N 5^{D N 2} ;$ Pdf $>C S N 5^{D N 4}$ ) or one copy of the tim-Gal4 driver $\left(\right.$ tim $>C S N 5 ;$ tim $>C S N 5^{D N 2} ;$ tim $\left.>C S N 5^{D N 4}\right)$. The final bar shows phase shifts for flies in which viability of CSN nnull mutants was rescued by expressing a wild-type UAS-CSN5 transgene using tubulinP-Gal4 and then preventing expression in LNs via Pdf-Gal8O (tub > CSN5, Pdf-Gal80, (SN $\left.5^{\text {null }}\right)$. Each bar represents a total of at least 18 flies analyzed in at least three independent experiments for phase advances (except 15 flies for tub $>$ CSN5, Pdf-Gal80, CSN5 $5^{\text {null }}$ ) and at least 10 for phase delays. Errorbars show SEM. Phase advances and phase delays were significantly reduced compared with controls with UAS-CSN5 $5^{D N 2}$ or UAS-CSN5 ${ }^{D N 4}$ transgenes expressed using either driver and also with tub $>$ CSN5, Pdf-Gal80, CSN5 ${ }^{\text {null }}$ flies $(p<0.001) . B$, Pdf $>+$ control flies and Pdf $>C S N 5^{D N 2}$ flies were entrained to LD cycles and then either dissected at ZT22 (top panels) or after 2 min light at ZT21 and an additional hour of darkness (bottom panels). Brains were stained with antibodies to TIM (red) and PDF (green). C, Quantitation of TIM levels in Pdf $>+$ control flies and Pdf $>$ CSN5 ${ }^{\text {DN2 }}$ flies at ZT22 or after a 2 min light pulse at ZT21 followed by $1 \mathrm{~h}$ darkness. The data are an average of three independent experiments with five to eight brains per experiment. The amount of TIM remaining after light is statistically lower in $P d f>+$ control flies than in $P d f>C \mathrm{CN}^{D N 2}$ flies $(p<0.01)$. We came to the same conclusions comparing these genotypes with a 2 min light pulse starting at $Z T 15(p<0.01)$ and also comparing Pdf $>+$ with $P d f>C S N 5^{D N 4}$ flies at each time point $(p<0.01)$ (data not shown).

\section{CSN4 and CSN5 are required for light-mediated TIM degradation in larval LNs}

The persistence of TIM in the early morning after the light onset suggested that CSN mutants have reduced responses to light. Thus, we tested whether the CSN is required for light-dependent
TIM degradation in LNs. Larvae were given either a $2 \mathrm{~h}$ light pulse starting at ZT15 when TIM is in the cytoplasm, or a $1 \mathrm{~h}$ light pulse starting at ZT23 when TIM is nuclear. TIM levels after light exposure were quantified and compared with TIM levels in unpulsed larvae at ZT17 or ZT24, respectively. The results in Figure 2 show that TIM is mostly degraded in wild-type larval LNs after light pulses at ZT15 and ZT23 with only 5-15\% TIM remaining. In contrast, $70-80 \%$ of TIM remained in CSN4 $4^{\text {null }}$ and CSN5 $5^{\text {null }}$ larval LNs after the light pulses. We conclude that an intact CSN is required for the normal lightmediated degradation of both cytoplasmic and nuclear TIM in larval LNs.

CSN5 is required cell autonomously for light-dependent TIM degradation in LNs

Because the CSN4 $4^{\text {null }}$ and CSN5 $5^{\text {null }}$ mutations affect the entire larva, our results could be explained by the mutations affecting, for example, visual system signaling to LNs that could in turn affect TIM degradation. CSN5 was previously shown to be important in Drosophila photoreceptor differentiation and glial cell migration in the optic lobe (Suh et al., 2002). However, Bolwig's organ, the simple larval visual system, appeared structurally normal in CSN $4^{\text {null }}$ and CSN $5^{\text {null }}$ mutants and projected to the LNs as it does in wild-type larvae (A. Knowles, S. Sprecher, J. Blau, unpublished observations).

To test whether the CSN is required for TIM degradation in a cell-autonomous manner, we used the Gal4-UAS system to engineer mosaic larvae with altered CSN activity only in LNs. Ideally, these experiments would consist of (1) LN-specific rescue of a CSN subunit null mutation and (2) expression of a dominant-negative CSN subunit specifically in LNs. We chose CSN5 for these experiments because a single amino acid change ( $\mathrm{D}$ to $\mathrm{N}$ at position 148) abolishes CSN5 deneddylase activity, but this mutant CSN5 (CSN5 ${ }^{\mathrm{D} 148 \mathrm{~N}}$, referred hereafter to as $\mathrm{CSN} 5^{\mathrm{DN}}$ ) is still incorporated into the CSN complex but inhibits CSN activity (Cope et al., 2002; Wu et al., 2005).

Wild-type CSN5 expression was restored specifically to LNs using a Pdf-Gal4 driver to express a UAS-CSN5 $5^{W T}$ transgene in a $C S N 5^{\text {null }}$ background. Light pulse experiments beginning at ZT15 and ZT23 were performed as outlined above to test for degradation of cytoplasmic and nuclear TIM, respectively. The results in Figure 3 show that TIM degradation in wild-type larvae was indistinguishable from larvae in which CSN5 expression was restored to LNs. In contrast, $C S N 5^{\text {null }}$ mutant larvae had minimal TIM degrada- 


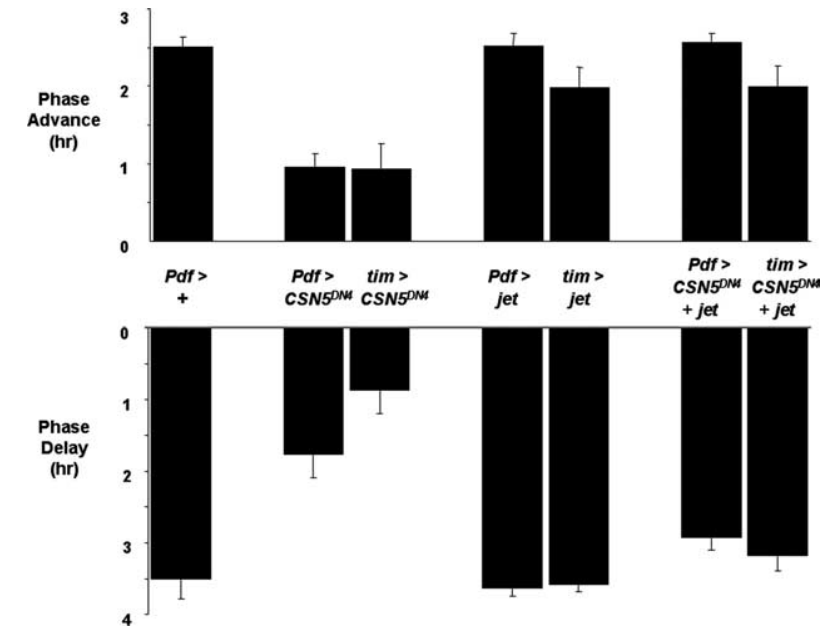

Figure 6. Overexpression of jet in clock neurons suppresses the defects in phase shifts to light of flies with dominant-negative CSN5. Phase shifts were measured and plotted as in Figure 5. Either $P d f_{0.5}$-Gal4, with two copies of the driver, or tim-Gal4 was used to express UAS-CSN5 ${ }^{D N 4}$ alone, UAS-jet alone, or both transgenes together. The data for $P d f>+, P d f>C S N 5^{D N 4}$ and tim $>$ CSN $5^{D N 4}$ are replotted from Figure 5 . For the new genotypes, each bar represents at least 15 flies analyzed in at least two independent experiments. Overexpression of jet via $P d f_{0.5}$-Gal4 or tim-Gal4 has no effect on phase shifts compared with controls $(p>0.15)$. Phase shifts are reduced in $P d f>C S N 5^{D N 4}$ flies compared with $P d f>C S N 5^{D N 4}+$ jet flies $(p<0.0001)$ and between tim $>\operatorname{CSN} 5^{D N 4}$ flies and tim $>\operatorname{CSN} 5^{D N 4}+$ jet flies $(p<0.003)$.

tion. Thus, CSN5 is required within LNs for normal lightdependent TIM degradation.

In the complementary experiment, we used Pdf-Gal4 to express a dominant-negative $U A S-C S N 5^{D N}$ transgene in an otherwise wild-type background and repeated the light pulse experiments as outlined above. We found that TIM degradation in larval LNs was mostly blocked by two independent $U A S-C S N 5^{D N}$ lines [DN2 (Fig. 4) and DN4 (data not shown)]. There is partial inhibition of TIM degradation with the UAS-CSN5 ${ }^{D N 2}$ transgene even in the absence of a driver (Fig. $4 A$ ), suggesting slightly leaky expression of the UAS-CSN $5^{D N 2}$ transgene. However, TIM degradation in LNs is potently inhibited in larvae with Pdf-Gal4 and $U A S-C S N 5^{D N 2}$ and TIM levels after light in these mosaic larvae are comparable with those observed in $C S N 5^{\text {null }}$ mutant larvae. Thus, expression of the UAS-CSN5 ${ }^{D N}$ transgene in LNs is sufficient to block TIM degradation.

\section{CSN5 is required for resetting adult behavioral rhythms}

TIM degradation in response to light resets the molecular clock, and this entrains and shifts the phase of behavioral rhythms of adult flies. Thus, if inhibiting CSN activity prevents lightdependent TIM degradation, then this should also cause defects in resetting the phase of adult behavioral rhythms via light. To test this prediction, we used the UAS-CSN5 ${ }^{D N}$ transgenes described above to alter CSN activity specifically in clock neurons and then measured the behavioral responses of flies to light.

First, we checked whether expression of UAS-CSN5 ${ }^{W T}$ or either UAS-CSN5 ${ }^{D N}$ line with Pdf-Gal4 $\left(\mathrm{LN}_{\mathrm{v}} \mathrm{s}\right.$ only) or tim-Gal4 (all clock neurons) altered circadian rhythms of adult locomotor activity rhythms in DD. The results in Table 1 show that the period length and strength of these rhythms was mostly unaffected by these manipulations. Therefore, CSN5 activity is not essential for adult circadian rhythms, consistent with the oscillations of TIM protein in CSN5 $5^{\text {null }}$ larval LNs (Fig. 1).

Exposure to light either delays or advances the phase of adult behavioral rhythms on subsequent days by triggering TIM deg- radation. Light received by flies in the early part of the night (e.g., ZT15) delays the phase of the behavioral rhythm, whereas light pulses in the late night (e.g., ZT21) advance the phase. At the molecular level, behavioral phase delays correlate with degradation of cytoplasmic TIM delaying its accumulation, nuclear entry, and subsequent steps of the cycle. Conversely, behavioral phase advances are correlated with degradation of nuclear TIM that leads to earlier reactivation of per and tim expression by prematurely removing repression by PER and TIM.

To assess how light-dependent TIM degradation affects behavioral phases, we compared the phase shifts of adult flies expressing either $U A S-C S N 5^{W T}$ or $U A S-C S N 5^{D N}$. For these experiments, flies entrained in LD cycles were given a 2 min light pulse at either ZT15 or ZT21 and were then moved to DD. We calculated the average time at which flies stopped activity on each of the first $4 \mathrm{~d}$ in DD and compared it with LD-entrained flies that were moved to DD without a light pulse (Fig. 5A).

Expression of wild-type UAS-CSN5 either only in $\mathrm{LN}_{\mathrm{v}} \mathrm{s}$ or in all clock cells had minimal effects on phase shifts compared with control Pdf-Gal4 flies (Fig. 5A). In contrast, expression of the dominant-negative $U A S-C S N 5^{D N}$ transgenes significantly reduced phase advances and delays (Fig. 5A). Phase shifts were inhibited slightly more with the tim-Gal4 driver than with $P d f$ Gal4, perhaps because the tim-Gal4 driver is also expressed in the Evening (E) clock neurons, which are important mediators of light entrainment (Stoleru et al., 2007). The residual phase shifts observed in flies expressing UAS-CSN5 ${ }^{D N}$ transgenes are consistent with low levels of TIM degradation seen in Figure 4. Overall, these results correlate well with the requirement for CSN activity for TIM degradation in larval LNs.

To test whether these behavioral defects in phase shifts of adult flies are attributable to impaired TIM degradation, we compared TIM degradation in control flies $(P d f>+)$ with flies lacking normal CSN5 activity in PDF cells $\left(P d f>C S N 5^{D N 2}\right)$. Flies were exposed to $2 \mathrm{~min}$ of light at either ZT15 or ZT21 as in the phase shift experiments, and then TIM levels were measured $1 \mathrm{~h}$ later. The results for ZT21 are shown in Figure 5, $B$ and $C$, and reveal extensive TIM degradation in control flies, but minimal TIM degradation in $P d f>C S N 5^{D N 2}$ flies. Similar defects in TIM degradation were seen when a 2 min light pulse was given at ZT15, and also in $P d f>C S N 5^{D N 4}$ flies at both time points (data not shown). These data support the idea that flies lacking normal CSN5 activity in clock neurons show defective phase shifts because they cannot degrade TIM normally.

We also generated flies in which the lethality of the CSN5 $5^{\text {null }}$ mutation was rescued by expressing a wild-type UAS-CSN5 transgene throughout the fly using tubulinP-Gal4 and then inhibiting Gal4 activity specifically in $\mathrm{LN}_{\mathrm{v}} \mathrm{s}$ via a Pdf-Gal80 transgene. The period length and strength of their locomotor rhythms in DD were indistinguishable from those of wild-type flies (Table 1). This further supports our conclusion that wild-type CSN activity in $\mathrm{LN}_{\mathrm{v}} \mathrm{s}$ is not required for normal circadian rhythms in DD.

Next, we measured the magnitude of phase delays and advances in response to light pulses at ZT15 and ZT21 as described above. We found that the phase of these flies was almost unchanged after a light pulse (Fig. 5A). These results support the idea that the CSN acts cell autonomously within $\mathrm{LN}_{\mathrm{v}} \mathrm{s}$ and is required for clock resetting, presumably by mediating TIM degradation in $\mathrm{LN}_{\mathrm{v}} \mathrm{s}$.

\section{CSN5 and JET cooperate for clock resetting by light}

The deneddylation activity of the CSN has been proposed to inactivate SCF complexes. Thus, a nonfunctional CSN might be 
A
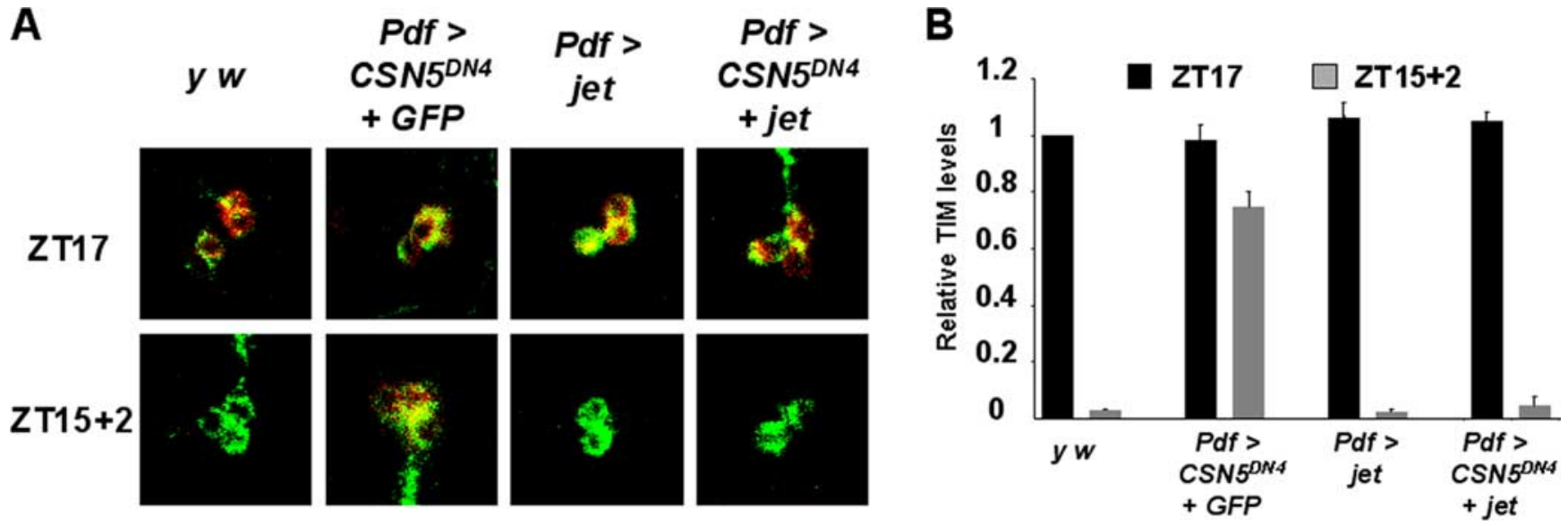

C

$\mathrm{ZT} 15+2$ CSN5 DN4

Pdf > CSN5 DN4
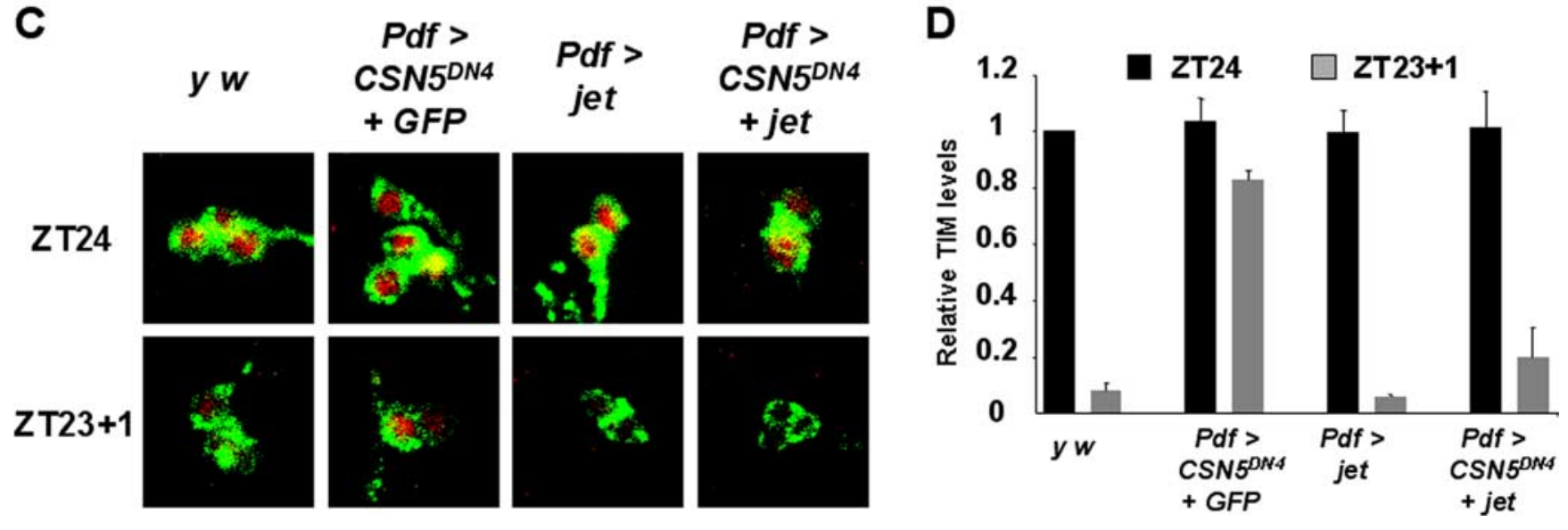

Figure 7. Overexpression of JET in larval LNs rescues defects in light-mediated TIM degradation caused by dominant-negative CSN5. Light pulse experiments were performed and quantitated as in Figure 2 with light pulses starting at ZT15 $(\boldsymbol{A}, \boldsymbol{B})$ or ZT23 (C, D). TIM is shown in red, and PDF is shown in green. Larvae used were y $w$ controls (left panels), or Pdf ${ }_{0.5}^{-G a l 4}$ driving expression of UAS-CSN5 ${ }^{D N}$ and UAS-CD8::GFP transgenes (Pdf $>$ CSN5 ${ }^{D N 4}+$ GFP), UAS-Jet alone (Pdf $>$ jet), or UAS-CSN5 ${ }^{D N}$ and UAS-Jet (Pdf $>$ CSN5 ${ }^{D N 4}+$ jet). The images on the left are representative of at least 10 brains stained per time point in at least four independent experiments. Quantitation was performed for three of these experiments using at least five brains per genotype. Error bars show SEM. Levels of TIM degradation by light in $y w$ and Pdf $>$ jet larvae are similar $(p>0.1)$ TIM is degraded significantly better in Pdf $>C S N 5^{D N 4}+$ jet larvae than in Pdf $>$ CSN5 ${ }^{D N 4}+G F P$ larvae $(p<$ 0.001).

expected to lead to constitutively active SCF ${ }^{\mathrm{IET}}$ complexes and increased TIM degradation. However, in Neurospora csn-2 null mutants, the F-box protein FWD-1 was found to be highly unstable while its substrate, the clock protein FRQ, overaccumulated (He et al., 2005). Thus, the absence of CSN activity seems to lead to constitutively active SCF complexes resulting in the degradation or inactivation of the F-box protein itself, and thus allowing substrate accumulation. This model would explain our results: in the absence of CSN activity, constitutively active $\mathrm{SCF}^{\mathrm{JET}}$ complexes lead to degradation or inactivation of JET itself, thus preventing TIM degradation after a light pulse. In this scenario, JET levels or activity would be limiting for TIM degradation when CSN activity is inhibited, and therefore increased jet expression might rescue defects in TIM degradation when CSN activity is inhibited via $U A S-C S N 5^{D N}$.

To test this idea, we used flies in which Pdf-Gal4 was used to express UAS-jet and UAS-CSN5 ${ }^{D N 4}$ simultaneously. As a preliminary experiment, we first measured phase shifts in adult flies in which Pdf-Gal4 was used to express UAS-jet. We found that the magnitude of phase delays and advances was similar to controls (Fig. 6). Next, we tested the phase shifts of flies in which Pdf-Gal4 was used to coexpress UAS-jet and UAS-CSN5 ${ }^{D N 4}$ simultaneously. Phase shifts of these flies were essentially indistinguishable from flies expressing UAS-jet alone. Thus, increasing JET levels in LNs rescues the defective clock resetting of flies expressing UAS-CSN5 ${ }^{D N 4}$ (Fig. 6). This result is not a trivial effect of dividing Gal4 activity between two UAS transgenes because flies expressing both UAS-CSN5 $5^{D N 4}$ and UAS-CD8::GFP in LNs showed similar defects in clock resetting to flies expressing the $U A S-C S N 5^{D N 4}$ transgene alone (data not shown).

CSN5 and JET cooperate for TIM degradation by light

Because the $C S N 5^{D N}$ clock-resetting defect could be rescued by overexpressing jet, it seemed likely that TIM degradation was also restored. We tested this by measuring TIM degradation in larval LNs. First, we measured TIM degradation in larvae overexpressing jet in LNs and found that TIM was degraded very well in this genetic background (Fig. 7). Next, we measured TIM degradation in larvae in which Pdf-Gal4 was used to express UAS$C S N 5^{D N 4}$ and UAS-jet transgenes simultaneously. The results show that TIM degradation in the early evening (ZT15) and late night (ZT23) was restored to levels very close to wild-type controls (Fig. 7). Again, this result is not a trivial effect of diluting Gal4 activity between two transgenes because larvae expressing both UAS-CSN5 $5^{D N 4}$ and UAS-CD8::GFP in LNs showed as high levels of TIM after light as the single UAS-CSN5 ${ }^{D N}$ transgene experiments shown in Figure 4. Thus, increased levels of JET protein override the defects in TIM degradation associated with 


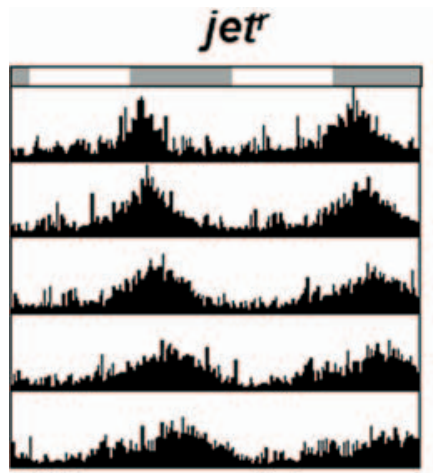

tim > CSN5DN

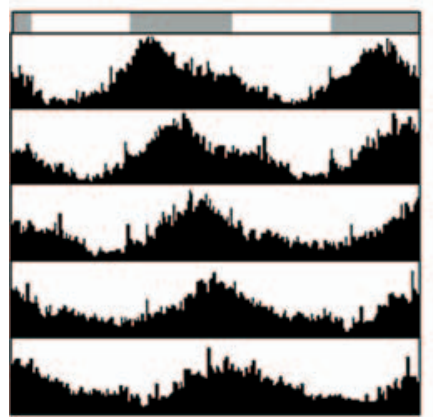

$+>$ CSN5 $^{\mathrm{DN}}$

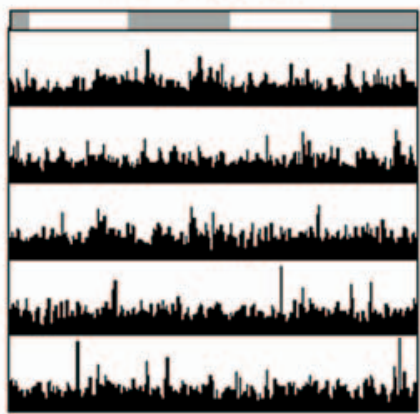

$P d f>C S N 5^{D N}$

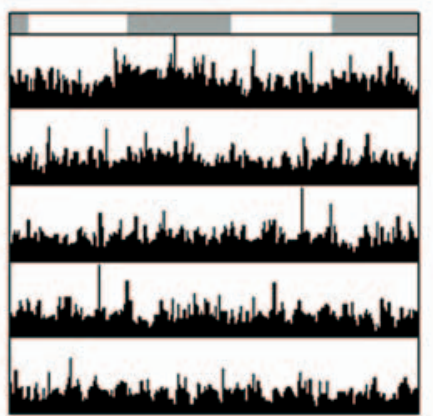

tim > +

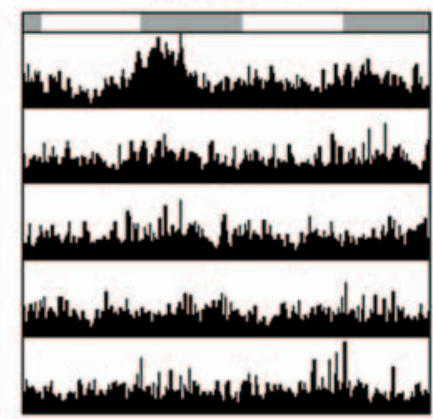

tim; Pdf80 > CSN5DN

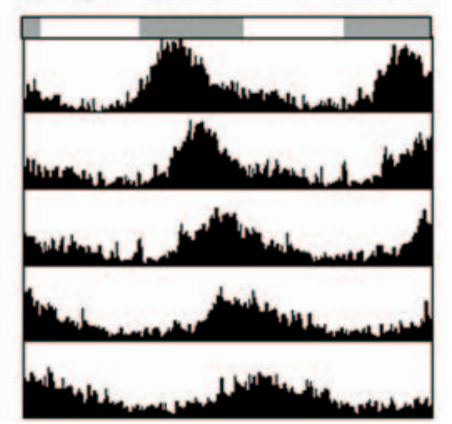

Figure 8. Inhibiting CSN activity in adult clock neurons makes flies rhythmic in constant light. Flies were entrained to LD cycles for $2-3 \mathrm{~d}$, and then moved to 100 lux constant light and their locomotor activity was recorded for $6 \mathrm{~d}$. The actograms are combined from all the flies tested (ranging between 26 and 44 flies depending on the genotype) and were from between one and three separate experiments. The different genotypes shown are as follows: top row, jet ${ }^{r}$ mutants (left), and $+/ U A S-C S N 5^{D N 2}$ ( $+>$ CSN5 ${ }^{\text {DN }}$; middle), and tim-gal4/ + control flies (tim $>+;$ right); bottom row, UAS-CSN5 $5^{D N 2}$ expressed via tim-gal4 (tim $>$ CSN5 $5^{D N}$; left), Pdf $0.5^{-G a l 4}$ (Pdf $>$ CSN5 ${ }^{D N}$; middle), or via tim-gal4 with a Pdf-Gal80 transgene (tim; Pdf80 $>$ CSN5 ${ }^{D N}$; right).

reducing CSN activity. We have not been able to examine whether JET levels are reduced in pacemaker neurons when CSN activity is inhibited, because the available antibodies to JET are not sensitive enough to recognize JET in clock neurons (Koh et al., 2006). Nevertheless, the rescue of $C S N 5^{D N}$ mutant phenotype by overexpression of JET supports the idea that JET and the CSN lie in the same pathway leading to TIM degradation specifically in response to light.

Inhibiting CSN activity in clock neurons induces constant light rhythmicity In addition to reduced phase shifts in response to light pulses, cry and jet mutants exhibit another light-dependent pheno-

type: rhythmicity in constant light (Emery et al., 2000; Koh et al., 2006). Given the many similarities in TIM degradation and clock resetting between flies mutant for cry, jet, or CSN subunits, we examined the rhythms of flies lacking normal CSN activity in LL (100 lux). Under these lighting conditions, jet ${ }^{r}$ mutants were rhythmic with an average period of $25.4 \mathrm{~h}$, whereas control $w^{1118}$ flies, tim-gal4 driver flies without a UAS transgene and UAS$C S N^{D N 2}$ transgenic flies without a driver were almost all arrhythmic (Fig. 8, Table 2). In contrast, we found that $t i m>C S N^{D N 2}$ flies were rhythmic in constant light. Thus, like cry and jet mutants, rhythmicity in constant light can be produced by inactivating the CSN, supporting the idea that the CSN works in the same pathway as CRY and JET to degrade TIM in light.
Table 2. Locomotor activity rhythms of UAS-CSN5 $5^{D N}$ transgenic flies in constant light

\begin{tabular}{|c|c|c|c|}
\hline Genotype & \% Rhythmic ( $n=$ total tested) & Period (h) \pm SEM & Power \pm SEM \\
\hline jet ${ }^{r}$ & $92(37)$ & $25.4 \pm 0.1$ & $537 \pm 21$ \\
\hline$w^{1118}$ & $6(16)$ & 22.3 & $344 \pm 10$ \\
\hline UAS-CSN5 $5^{D N 2} /+$ & $4(26)$ & 23.8 & $350 \pm 7$ \\
\hline tim-Gal4/+ & $3(35)$ & 25.4 & $351 \pm 6$ \\
\hline tim-Gal4 / + UAS-CSN5 $5^{\text {DN2 }} /+$ & $93(44)$ & $26.2 \pm 0.1$ & $679 \pm 26$ \\
\hline$+/+;$ Pdf-Gal4 $/+$ & $0(31)$ & n.a. & $359 \pm 6$ \\
\hline$+/+;$ Pdf-Gal4/UAS-CSN5 $5^{D N 2}$ & $0(30)$ & n.a. & $357 \pm 7$ \\
\hline tim-Gal4 / +;Pdf-Gal80 / + & $0(11)$ & n.a. & $365 \pm 11$ \\
\hline tim-Gal4 / +;Pdf-Gal80 /UAS-CSN5 $5^{\text {DN2 }}$ & $100(42)$ & $26.4 \pm 0.1$ & $665 \pm 24$ \\
\hline
\end{tabular}

The period for each fly across $6 \mathrm{~d}$ in LL was determined by $\chi^{2}$ analysis. Average period calculations used only rhythmic flies. Power was calculated using ClockLab with 5 min collection bins, and all flies were assigned a power value. For arrhythmic flies (no peak above the significance line), the power was measured at a local peak between 23 and $27 \mathrm{~h}$. A fly classified as arrhythmic typically gives a rhythm "power" of between 340 and 370 . A t test (unpaired, unequal variance) revealed the following significant differences for power: tim-Gal 4 + versus tim-Gal4 / + UAS-CSN5 $5^{D N 2} /+$ flies; UAS-CSN5 ${ }^{D N 2} /+$ versu tim-Gal4 / +;UAS-CSN5 DN2 / + flies; and tim-Gal4 / +;Pdf-Gal80 / + versus tim-Gal4 / +;Pdf-Gal80 /UAS-CSN5 ${ }^{D N 2}$ flies $(p<0.0001)$. There were not significant differences between Pdf-Gal4/+ and Pdf-Gal4/UAS-CSN5 $5^{\text {DN2 }}$ flies or between UAS-CSN5 $5^{D N 2} /+$ and Pdf-Gal4/UAS-CSN5 $5^{D N 2}$ flies $(p=0.78$ and 0.50 , respectively). n.a., Not applicable.

The central brain clock neurons have been functionally divided into two main groups. One group is the PDF-producing $\mathrm{LN}_{\mathrm{v}} \mathrm{s}$, which are the most important neurons for rhythmicity in DD (Renn et al., 1999). A second less well defined group of PDFnegative (PDF-ve) clock neurons includes a subset of DN1 dorsal neurons that has been associated with rhythmicity in constant light (Murad et al., 2007; Stoleru et al., 2007). To test in which clock neurons CSN activity is required to prevent rhythmicity in constant light, we restricted expression of the UAS-CSN ${ }^{D N 2}$ transgene using either $P d f$-Gal4 to target $\mathrm{LN}_{\mathrm{v}} \mathrm{s}$, or a combination of the tim-Gal4 driver and a Pdf-Gal80 transgene (Stoleru et al., 2004) to target the PDF-ve clock neurons. The results in Figure 8 and Table 2 show that flies are arrhythmic when $U A S-C S N^{D N 2}$ is 
expressed only in PDF-positive cells, but are rhythmic when $U A S-C S N^{D N 2}$ is expressed only in PDF-ve clock neurons. These findings are consistent with previous results demonstrating that the PDF-ve clock neurons are the major cellular substrate for rhythmicity in constant light (Murad et al., 2007; Picot et al., 2007; Stoleru et al., 2007).

\section{Discussion}

In this study, we identified a requirement for the CSN in lightmediated TIM degradation in Drosophila pacemaker neurons. First, we found that null mutations that affect the whole animal $\left(C S N 4^{\text {null }}\right.$ and $C S N 5^{\text {null }}$ ) (Fig. 2) inhibit TIM degradation in larval LNs in response to light. These effects are cell autonomous because restoration of CSN5 expression only to larval LNs rescued the defects in TIM degradation (Fig. 3), whereas expression of a dominant-negative CSN5 transgene specifically in larval LNs blocked TIM degradation as effectively as null mutations in CSN4 and CSN5 (Fig. 4).

TIM degradation by light in pacemaker neurons correlates with the ability to reset the phase of adult behavioral rhythms (Hunter-Ensor et al., 1996; Lee et al., 1996; Myers et al., 1996; Zeng et al., 1996). We found that $C S N 5^{D N}$ transgenes potently inhibited phase shifts of the clock in response to light, and mosaic animals lacking CSN5 expression only in adult $\mathrm{LN}_{\mathrm{v}} \mathrm{s}$ behaved similarly (Fig. 5). The role of the CSN appears limited to lightmediated TIM degradation because the molecular clock oscillates in $C S N 4^{\text {null }}$ and CSN5 ${ }^{\text {null }}$ mutants (Fig. 1), and expression of $C S N 5^{D N}$ in clock neurons had no effect on adult locomotor activity rhythms in DD (Table 1). This specificity of the CSN is surprising because (1) the CSN has been implicated in the degradation of many proteins that are degraded by the ubiquitin-proteasome system; and (2) blocking degradation of PER protein either by reducing the activity of Double-Time, the PER kinase tightly linked to PER stability, or Slimb, the PER F-box, leads to strong changes on the molecular clock (Price et al., 1998; Rothenfluh et al., 2000; Suri et al., 2000; Grima et al., 2002; Ko et al., 2002) (discussed further below).

The phenotypes of jet mutant flies (Koh et al., 2006) are very similar to those we described for flies with altered CSN activity: reduced phase shifts and TIM degradation in LNs, and behavioral rhythmicity in constant light. In addition, the phase shift and TIM degradation phenotypes of $C S N 5^{D N}$ mutants were rescued by overexpression of jet. These results suggest that JET and CSN are elements in a common pathway connecting photoreception by CRY to TIM degradation. In Drosophila, both the visual system and CRY participate in clock resetting in a partially redundant manner (Stanewsky et al., 1998; Helfrich-Förster et al., 2001). jet and $C S N 5^{D N}$ mutant flies, as well as cry mutants, still synchronize to LD cycles, indicating that clock resetting is not completely inhibited in these mutants. However, clock resetting is abolished in glass; cry double mutants, which lack all known visual input to the circadian clock (Helfrich-Förster et al., 2001), suggesting that resetting of the clock in cry mutants occurs through the visual system. Similarly, the residual light entrainment in jet and $C S N 5^{D N}$ mutants may be mediated by the visual system in a CRY-independent manner.

The restoration of phase shifts and TIM degradation by overexpressing JET in $\mathrm{LN}_{\mathrm{v}}$ s expressing $C S N 5^{D N}$ supports the idea that JET has become limiting in $C S N 5^{D N} \mathrm{LN}_{\mathrm{v}} \mathrm{s}$, thus making it hard to degrade TIM by light. If JET levels are normally limiting in wildtype $\mathrm{LN}_{\mathrm{v}} \mathrm{s}$, then it would explain why TIM degradation by light is sensitive to the manipulations of CSN activity performed here. However, the lack of CSN dependence on SCF ${ }^{\text {Slimb-mediated }}$ degradation of TIM in constant conditions is counterintuitive. The requirement of the CSN for SCF-mediated protein degradation is well established (Schwechheimer et al., 2002; Yang et al., 2002; He et al., 2005; Cope and Deshaies, 2006). One possibility is that Slimb is either more abundant or more stable in LNs than JET, leading to SCF ${ }^{\text {Slimb }}$ complexes being less sensitive to inactivating the CSN. Alternatively, CSN control of SCF activity may be selective in $\mathrm{LN}_{\mathrm{v}}$ s such that the CSN is required to regulate SCF ${ }^{\mathrm{JET}}$ but not $\mathrm{SCF}^{\text {Slimb }}$. Although the mechanism of such selectivity is unknown, it is intriguing to note that, in Drosophila, the CSN is necessary for $\mathrm{SCF}^{\mathrm{Ago}}$-mediated degradation of Cyclin E (Doronkin et al., 2003), but not the signal-dependent degradation of Cactus, which is dependent on SCF ${ }^{\text {Slimb }}$ (Harari-Steinberg et al., 2007).

In conclusion, we demonstrated that the CSN has a specific role in the Drosophila clock in mediating light-triggered TIM degradation to reset the clock. Arguably the most exciting unanswered question in fly clock resetting is how signals from the visual system and/or CRY mark TIM for recognition by JET to trigger TIM degradation. Although a tyrosine kinase pathway was predicted based on inhibitor studies many years ago (Naidoo et al., 1999), no specific TIM kinases have yet been found. In mammalian cells, the CSN coordinates substrate-specific kinases (such as those for p53 and cJun) and substrate degradation (for review, see Harari-Steinberg and Chamovitz, 2004). Conceivably, a TIM-specific kinase could also interact with the CSN to regulate degradation of TIM. With the identification of such a kinase, we will have a detailed understanding of the biochemistry of clock resetting in Drosophila.

\section{References}

Allada R, Emery P, Takahashi JS, Rosbash M (2001) Stopping time: the genetics of fly and mouse circadian clocks. Annu Rev Neurosci 24:1091-1119.

Busino L, Bassermann F, Maiolica A, Lee C, Nolan PM, Godinho SI, Draetta GF, Pagano M (2007) SCF ${ }^{\text {Fbxl3 }}$ controls the oscillation of the circadian clock by directing the degradation of Cryptochrome proteins. Science 316:900-904

Chamovitz DA, Wei N, Osterlund MT, von Arnim AG, Staub JM, Matsui M, Deng XW (1996) The COP9 complex, a novel multisubunit nuclear regulator involved in light control of a plant developmental switch. Cell 86:115-121.

Cope GA, Deshaies RJ (2006) Targeted silencing of Jab1/Csn5 in human cells downregulates SCF activity through reduction of F-box protein levels. BMC Biochem 7:1.

Cope GA, Suh GS, Aravind L, Schwarz SE, Zipursky SL, Koonin EV, Deshaies RJ (2002) Role of predicted metalloprotease motif of Jab1/Csn5 in cleavage of Nedd8 from Cul1. Science 298:608-611.

Cyran SA, Yiannoulos G, Buchsbaum AM, Saez L, Young MW, Blau J (2005) The Double-Time protein kinase regulates the subcellular localization of the Drosophila clock protein Period. J Neurosci 25:5430-5437.

Deng XW, Dubiel W, Wei N, Hofmann K, Mundt K, Colicelli J, Kato J, Naumann M, Segal D, Seeger M, Carr A, Glickman M, Chamovitz DA (2000) Unified nomenclature for the COP9 signalosome and its subunits: an essential regulator of development. Trends Genet 16:202-203.

Doronkin S, Djagaeva I, Beckendorf SK (2003) The COP9 signalosome promotes degradation of Cyclin E during early Drosophila oogenesis. Dev Cell 4:699-710.

Emery P, So WV, Kaneko M, Hall JC, Rosbash M (1998) CRY, a Drosophila clock and light-regulated cryptochrome, is a major contributor to circadian rhythm resetting and photosensitivity. Cell 95:669-679.

Emery P, Stanewsky R, Hall JC, Rosbash M (2000) A unique circadianrhythm photoreceptor. Nature 404:456-457.

Freilich S, Oron E, Kapp Y, Nevo-Caspi Y, Orgad S, Segal D, Chamovitz DA (1999) The COP9 signalosome is essential for development of Drosophila melanogaster. Curr Biol 9:1187-1190.

Godinho SI, Maywood ES, Shaw L, Tucci V, Barnard AR, Busino L, Pagano M, Kendall R, Quwailid MM, Romero MR, O’neill J, Chesham JE, 
Brooker D, Lalanne Z, Hastings MH, Nolan PM (2007) The after-hours mutant reveals a role for Fbxl3 in determining mammalian circadian period. Science 316:897-900.

Grima B, Lamouroux A, Chélot E, Papin C, Limbourg-Bouchon B, Rouyer F (2002) The F-box protein Slimb controls the levels of clock proteins Period and Timeless. Nature 420:178-182.

Harari-Steinberg O, Chamovitz DA (2004) The COP9 signalosome: mediating between kinase signaling and protein degradation. Curr Protein Pept Sci 5:185-189.

Harari-Steinberg O, Ohad I, Chamovitz DA (2001) Dissection of the light signal transduction pathways regulating the two early light-induced protein genes in Arabidopsis. Plant Physiol 127:986-997.

Harari-Steinberg O, Cantera R, Denti S, Bianchi E, Oron E, Segal D, Chamovitz DA (2007) COP9 signalosome subunit 5 (CSN5/Jab1) regulates the development of the Drosophila immune system: effects on Cactus, Dorsal and hematopoiesis. Genes Cells 12:183-195.

Hardin PE (2005) The circadian timekeeping system of Drosophila. Curr Biol 15:R714-R722.

He Q, Cheng P, Yang Y, He Q, Yu H, Liu Y (2003) FWD1-mediated degradation of FREQUENCY in Neurospora establishes a conserved mechanism for circadian clock regulation. EMBO J 22:4421-4430.

He Q, Cheng P, He Q, Liu Y (2005) The COP9 signalosome regulates the Neurospora circadian clock by controlling the stability of the $\mathrm{SCF}^{\mathrm{FWD}-1}$ complex. Genes Dev 19:1518-1531.

Helfrich-Förster C, Winter C, Hofbauer A, Hall JC, Stanewsky R (2001) The circadian clock of fruit flies is blind after elimination of all known photoreceptors. Neuron 30:249-261.

Hunter-Ensor M, Ousley A, Sehgal A (1996) Regulation of the Drosophila protein timeless suggests a mechanism for resetting the circadian clock by light. Cell 84:677-685.

Ko HW, Jiang J, Edery I (2002) Role for Slimb in the degradation of Drosophila Period protein phosphorylated by Doubletime. Nature 420:673-678.

Koh K, Zheng X, Sehgal A (2006) JETLAG resets the Drosophila circadian clock by promoting light-induced degradation of TIMELESS. Science 312:1809-1812.

Lee C, Parikh V, Itsukaichi T, Bae K, Edery I (1996) Resetting the Drosophila clock by photic regulation of PER and a PER-TIM complex. Science 271:1740-1744.

Lee T, Luo L (1999) Mosaic analysis with a repressible cell marker for studies of gene function in neuronal morphogenesis. Neuron 22:451-461.

Lyapina S, Cope G, Shevchenko A, Serino G, Tsuge T, Zhou C, Wolf DA, Wei N, Shevchenko A, Deshaies RJ (2001) Promotion of NEDD-CUL1 conjugate cleavage by COP9 signalosome. Science 292:1382-1385.

Mazzoni EO, Desplan C, Blau J (2005) Circadian pacemaker neurons transmit and modulate visual information to control a rapid behavioral response. Neuron 45:293-300.

Murad A, Emery-Le M, Emery P (2007) A subset of dorsal neurons modulates circadian behavior and light responses in Drosophila. Neuron 53:689-701.

Myers MP, Wager-Smith K, Rothenfluh-Hilfiker A, Young MW (1996) Light-induced degradation of TIMELESS and entrainment of the Drosophila circadian clock. Science 271:1736-1740.

Naidoo N, Song W, Hunter-Ensor M, Sehgal A (1999) A role for the proteasome in the light response of the Timeless clock protein. Science 285:1737-1741.

Oron E, Mannervik M, Rencus S, Harari-Steinberg O, Neuman-Silberberg S, Segal D, Chamovitz DA (2002) COP9 signalosome subunits 4 and 5 regulate multiple pleiotropic pathways in Drosophila melanogaster. Development 129:4399-4409.

Oron E, Tuller T, Li L, Rozovsky N, Yekutieli D, Rencus-Lazar S, Segal D,
Chor B, Edgar BA, Chamovitz DA (2007) Genomic analysis of COP9 signalosome function in Drosophila melanogaster reveals a role in temporal regulation of gene expression. Mol Syst Biol 3:108.

Osterlund MT, Hardtke CS, Wei N, Deng XW (2000) Targeted destabilization of HY5 during light-regulated development of Arabidopsis. Nature 405:462-466.

Park JH, Helfrich-Förster C, Lee G, Liu L, Rosbash M, Hall JC (2000) Differential regulation of circadian pacemaker output by separate clock genes in Drosophila. Proc Natl Acad Sci U S A 97:3608-3613.

Peschel N, Veleri S, Stanewsky R (2006) Veela defines a molecular link between Cryptochrome and Timeless in the light-input pathway to Drosophila's circadian clock. Proc Natl Acad Sci U S A 103:17313-17318.

Picot M, Cusumano P, Klarsfeld A, Ueda R, Rouyer F (2007) Light activates output from evening neurons and inhibits output from morning neurons in the Drosophila circadian clock. PLoS Biol 5:2513-2521.

Price JL, Blau J, Rothenfluh A, Abodeely M, Kloss B, Young MW (1998) double-time is a novel Drosophila clock gene that regulates PERIOD protein accumulation. Cell 94:83-95.

Renn SC, Park JH, Rosbash M, Hall JC, Taghert PH (1999) A pdf neuropeptide gene mutation and ablation of PDF neurons each cause severe abnormalities of behavioral circadian rhythms in Drosophila. Cell 99:791-802.

Rothenfluh A, Abodeely M, Young MW (2000) Short-period mutations of per affect a double-time-dependent step in the Drosophila circadian clock. Curr Biol 10:1399-1402.

Schwechheimer C, Serino G, Deng XW (2002) Multiple ubiquitin ligasemediated processes require COP9 signalosome and AXR1 function. Plant Cell 14:2553-2563.

Siepka SM, Yoo SH, Park J, Song W, Kumar V, Hu Y, Lee C, Takahashi JS (2007) Circadian mutant Overtime reveals F-box protein FBXL3 regulation of cryptochrome and period gene expression. Cell 129:1011-1023.

Stanewsky R, Kaneko M, Emery P, Beretta B, Wager-Smith K, Kay SA, Rosbash M, Hall JC (1998) The $\mathrm{cry}^{b}$ mutation identifies Cryptochrome as a circadian photoreceptor in Drosophila. Cell 95:681-692.

Stoleru D, Peng Y, Agosto J, Rosbash M (2004) Coupled oscillators control morning and evening locomotor behaviour of Drosophila. Nature 431:862-868.

Stoleru D, Nawathean P, Fernández MP, Menet JS, Ceriani MF, Rosbash M (2007) The Drosophila circadian network is a seasonal timer. Cell 129:207-219.

Suh GS, Poeck B, Chouard T, Oron E, Segal D, Chamovitz DA, Zipursky SL (2002) Drosophila JAB1/CSN5 acts in photoreceptor cells to induce glial cells. Neuron 33:35-46.

Suri V, Hall JC, Rosbash M (2000) Two novel doubletime mutants alter circadian properties and eliminate the delay between RNA and protein in Drosophila. J Neurosci 20:7547-7555.

Wei N, Deng XW (2003) The COP9 signalosome. Annu Rev Cell Dev Biol 19:261-286.

Wei N, Chamovitz DA, Deng XW (1994) Arabidopsis COP9 is a component of a novel signaling complex mediating light control of development. Cell 78:117-124.

Wolf DA, Zhou C, Wee S (2003) The COP9 signalosome: an assembly and maintenance platform for cullin ubiquitin ligases? Nat Cell Biol 5:1029-1033.

Wu JT, Lin HC, Hu YC, Chien CT (2005) Neddylation and deneddylation regulate Cul1 and Cul3 protein accumulation. Nat Cell Biol 7:1014-1020.

Yang X, Menon S, Lykke-Andersen K, Tsuge T, Di Xiao, Wang X, RodriguezSuarez RJ, Zhang H, Wei N (2002) The COP9 signalosome inhibits p27(kip1) degradation and impedes $\mathrm{G}_{1}-\mathrm{S}$ phase progression via deneddylation of SCF Cul1. Curr Biol 12:667-672.

Zeng H, Qian Z, Myers MP, Rosbash M (1996) A light-entrainment mechanism for the Drosophila circadian clock. Nature 380:129-135. 